\title{
LA AGUJA DEL PALAU GÜELL
}

\section{(THE NEEDLE OF THE PALAU GÜELL)}

Josep Maria Moreno Lucas, Arquitecto Técnico

Servicio del Patrimonio Arquitectónico de la Diputación de Barcelona. España

\section{RESUMEN}

La bella aguja que emerge de la azotea del Palau Güell puede definirse, según el autor de este artículo, como una cúpula de concepción clásica con todos sus elementos. Se compone de tres partes esenciales: la cúpula - de estructura única (no doble como se habia dicho hasta ahora), formada por cuatro anillos superpuestos de pequeñas bóvedas parabólicas - la linterna - con ventanas de perfil parabólico y otras aberturas romboidales-, y la veleta-pararrayos, un conjunto escultórico decorativo y funcional hecho de hierro. latón y cobre.

En el artículo se analizan con detalle los aspectos constructivos, estáticos y formales del conjunto y cada una de sus partes. Un aspecto especialmente interesante es 10 referente al revestimiento exterior de la aguja, hecho con pequeños fragmentos de piedra arenisca vitrificada procedente de las caras interiores de los antiguos hornos de cal de leña amortizados. La naturaleza de este revestimiento. hasta ahora no estudiada, se publica por primera vez en estas páginas.

\section{SUMMARY}

The beautiful needle which emerges from the roof of the Palau Güell can be defined, according to the author of this article, as a cupola of classic conception with all its elements. It is composed of three essential parts: the cupola -of a single structure (and not double as had been said previous(y), made of four superimposed rings of small parabolic vaults, the lantern -with windows of a parabolic profile and other rhomboid apertures; and the weathercock and lightening rod, a decorative and functional sculptural group made of iron, brass and copper.

In this article, the constructive, static and formal aspects of the group and each one of the parts are analyzed in detail. An especially interesting aspect refers to the exterior facing of the needle, made of small pieces of glazed sandstone from the inside walls of the wood-burning time ovens, now abandoned. The nature of this facing, unstudied until now, is published for the first time in these pages.
La aguja cónica que corona el Palau Güell emerge 16 metros de la azotea (Fig. 1). Posee grupos de aberturas de diferentes tipologías y dimensiones, que cumplen funciones diversas, y un revestimiento singular hecho de pequeños fragmentos de piedra arenisca vidriada de forma fortuita.

La aguja se compone, tanto constructiva como compositivamente de tres partes bien definidas: la cúpula, la linterna y la veleta-pararrayos que remata el conjunto. Se puede definir, pues, a pesar de sus particulares formas y volúmenes, como una cúpula de concepción clásica con todos sus elementos: arcos, pechinas, contrafuertes, cúpula, linterna y veleta.

\section{LA CÚPULA}

La cúpula (Fig. 2) está formada por cuatro anillos con- céntricos superpuestos, que por el exterior forman una figura de perfil escalonado y por el interior definen una figura de revolución de generatriz parabólica. El anillo inferior se asienta en una circunferencia formada por la unión mediante pechinas de cuatro arcos parabólicos. Sobre el anillo superior se apoya un casquete es. férico que dispone de un óculo cenital que deja pasar al interior la luz captada a través de la linterna.

Cada uno de estos cuatro anillos está constituido por dieciséis pequeñas bóvedas parabólicas abocinadas, con la curvatura mayor hacia el exterior y la menor, por tanto, en el intradós de la cúpula. Estas bóvedas - son bóvedas, no arcos, ya que son elementos más largos en su profundidad que gruesos - están provistas en el punto medio de su longitud de un cerramiento fijo compuesto por marco de madera y vidrīo, pequeñas ventanas que dejan pasar la luz pero no el viento ni el agua. 
Informes de la Construcción, Vol. 42 n. ${ }^{\circ} 408$, julio/agosto, 1990

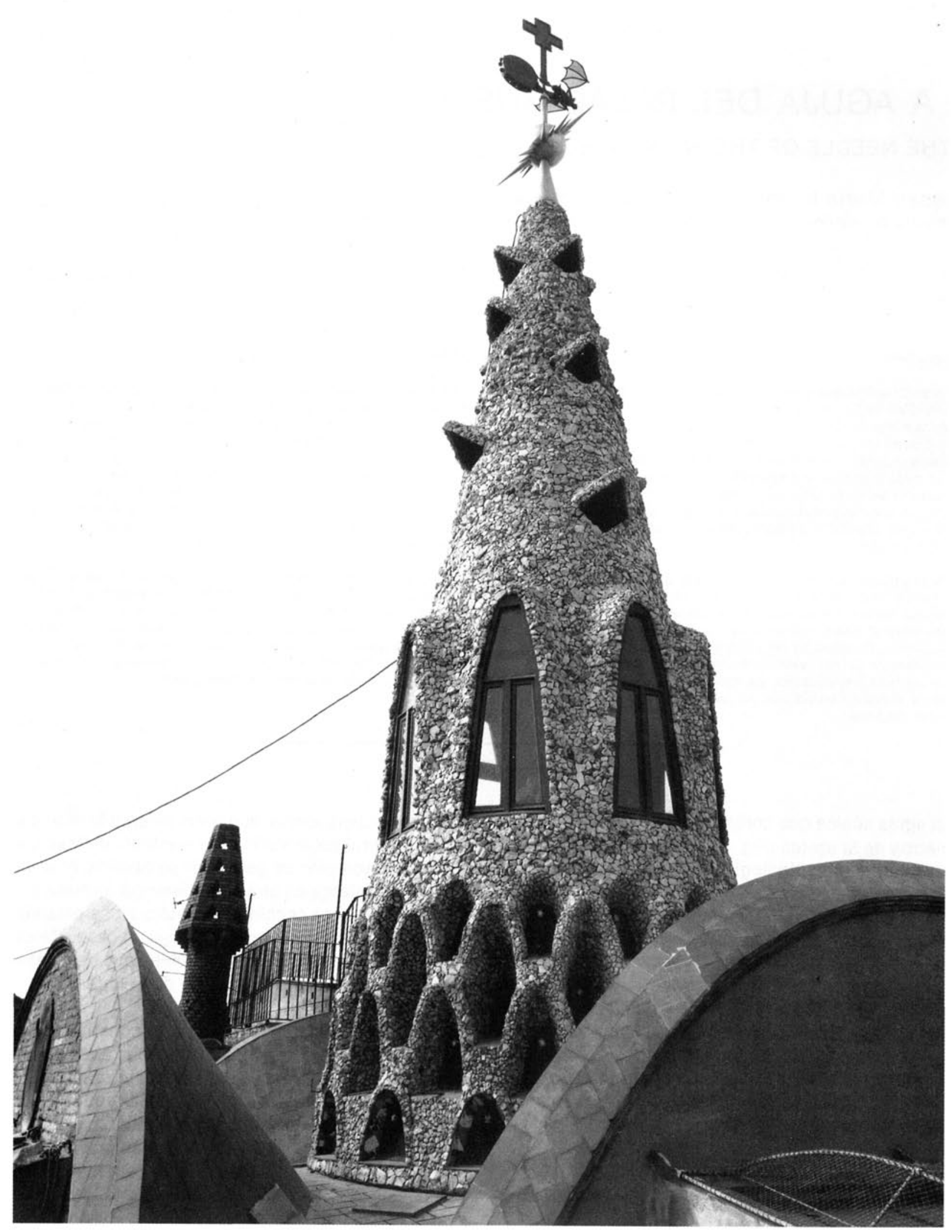

Fig. 1-La aguja poco después de la restauración. (Foto Jordi Isern, febrero de 1990). 
Internamente (Fig. 3) tanto la superficie de la cúpula como la de las pechinas en las que se asienta, está aplacada con piezas hexagonales de alabastro sanguino, tipo oriental, cuya dimensión disminuye respecto a la de la hilada inferior a fin de mantener el mismo número de piezas en cada hilada. Algunos hexágonos, de forma alternativa, disponen de orificios circulares que coinciden con las bóvedas de los anillos estructurales, que permiten la entrada de luz, desde el exterior.

Las pechinas y la cúpula definen una curvatura continua, de forma que seccionando el conjunto por la diagonal de la planta, se obtiene en alzado una parábola simétrica. Ello permite que el aspecto interior sea el de una bóveda a vela o vaida, si bien como se verá claramente al tratar del sistema constructivo, es una cúpula canónica formada por una parábola de revolución.

Salvando las distancias de todo tipo, especialmente en cuanto a sus formas volumétricas y en sus sistemas constructivos, se puede decir que en su concepto arquitectónico las cúpulas del Palau Güell y la del Panteón de Roma son similares. No se trata, naturalmente, de compararlas, sino de observar que la pequeña cúpula gaudiniana -con sus particulares formas arquitectónicas - está construida siguiendo los cánones clásicos con que se construyó aquélla.

En ambas, los anillos escalonados exteriores hacen de contrafuerte de las cargas que les transmite el casquete esférico superior, y así como en la cúpula del Panteón la estructura de los anillos escalonados está aligerada de peso mediante los casetones encofrados del interior, en la del Palau Güell se aligera mediante las pequeñas bóvedas abocinadas y superpuestas. Al ser la cúpula del Panteón una semiesfera perfecta, sus escalones exteriores son regulares en la relación alturaanchura. La del Palau Güell, al estar formada por una parábola de revolución, crea unos anillos escalonados cuya relación en altura es mayor que en anchura.

\section{Sistema constructivo}

Como hemos visto, la base de la cúpula se apoya sobre la circunferencia que forman el perfil superior de las pechinas. Desde los arcos parabólicos en los que descansan las pechinas - construidos con piedra caliza pulimentada del Garraf - por su parte posterior, y tangentes a ellos, arrancan otros arcos de ladrillo de forma escalonada cuyas impostas se apoyan sobre las diagonales del cuadrado que forma la figura geométrica donde se inscribe la base de la cúpula (ver fig. 4).

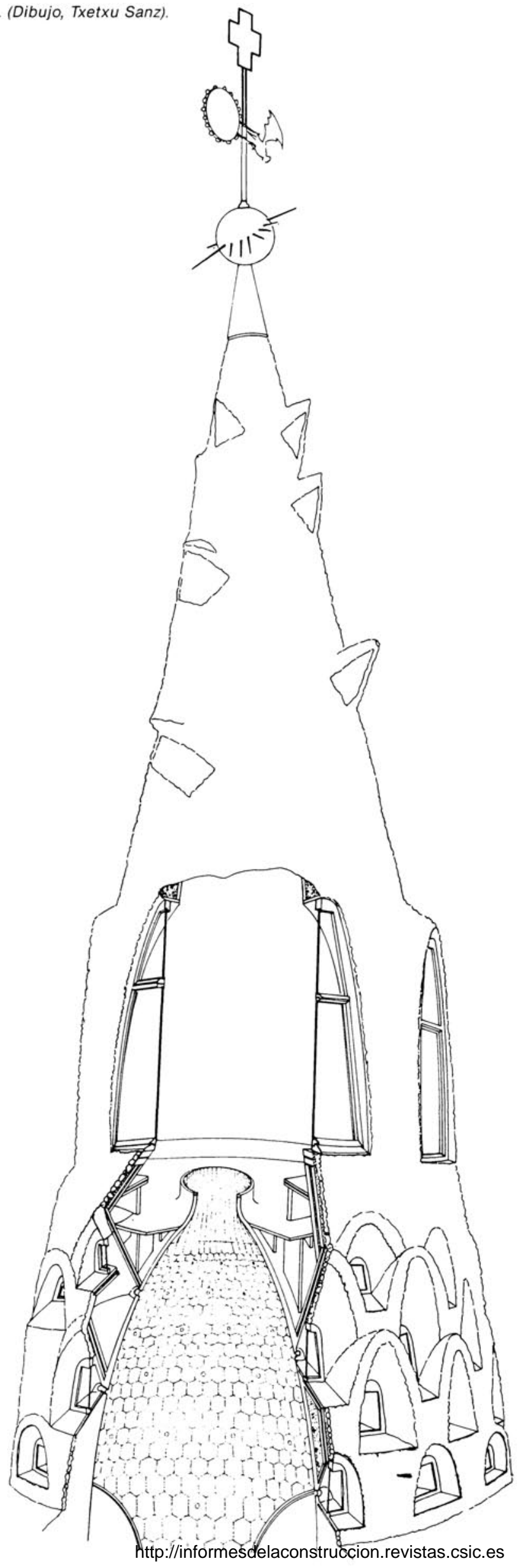




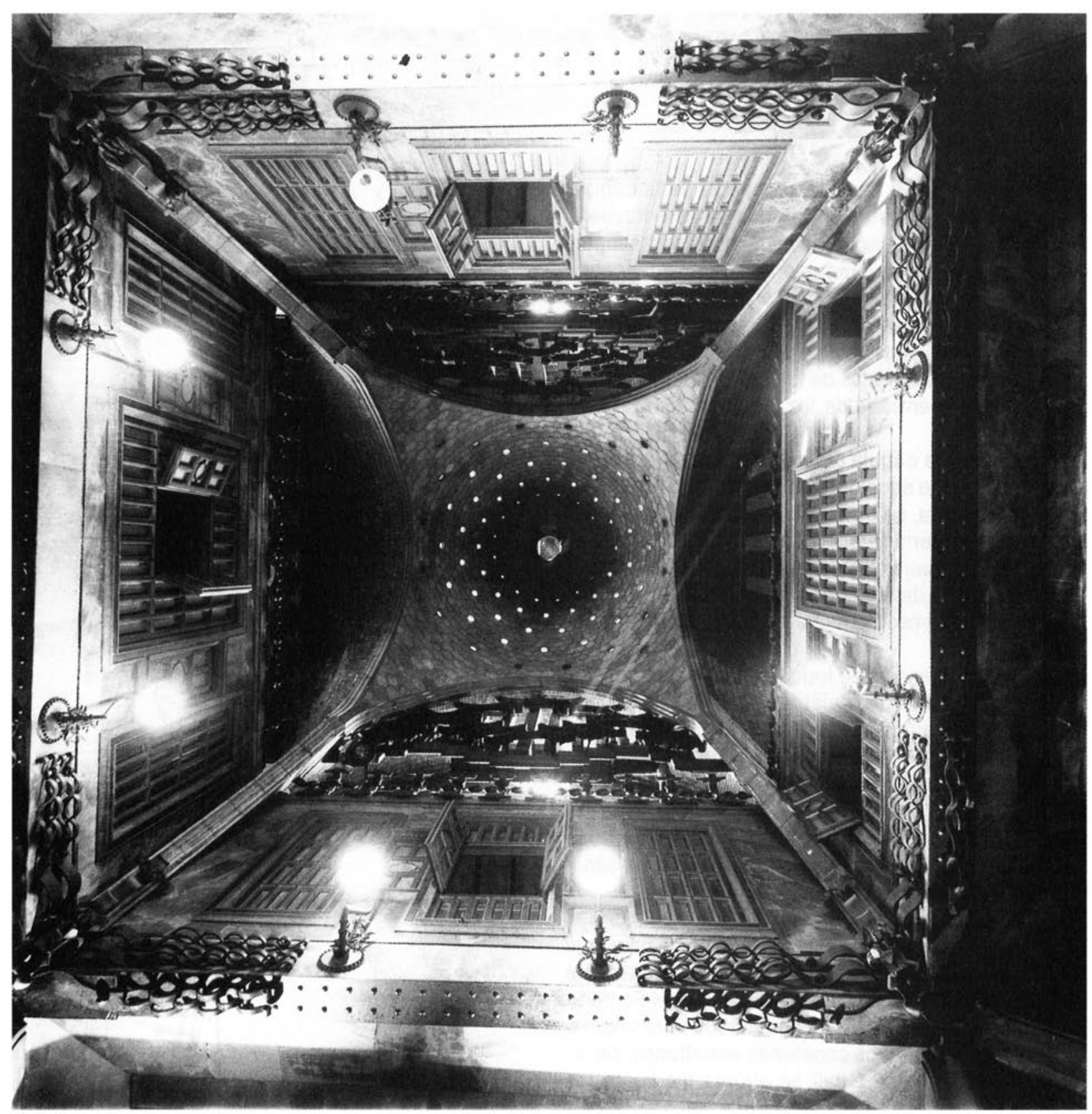

Fig. 3.-Espacio interior de la aguja desde el salón central de la planta noble. (Foto Joan Francés, junio de 1986).

Estos cuatro conjuntos de arcos cerámicos emergen en la azotea en forma de cuatro gajos cónicos de tal manera que el sector circular de las intersecciones verticales forman cuatro lunetas, las cuales a través de unos óculos circulares abiertos en su superficie, permiten la entrada de luz en el espacio interior de los arcos.

Las pechinas están construidas con ladrillo macizo, colocado a soga en la parte baja de las mismas. Conforme la curva se va pronunciando en la zona alta, el aparejo cambia a tizón, formando un pequeño escalonado por el intradós de la pechina que facilita la formación de la curva, mientras que por el extradós se mantiene vertical para crear en la parte alta una mayor superficie de apoyo de la base de la cúpula. En cada una de las pechinas hay tres pequeñas aberturas cuadradas, que coinciden con los hexágonos perforados del revestimiento, para permitir el paso de la luz a través de cuatro grupos de tres pequeñas claraboyas transitables, construidas en el pavimento de la azotea en la zona situada entre las lunetas. Las pequeñas bóvedas de los anillos que forman la cúpula están construidas con dos gruesos de rasilla común tomadas con mortero de cal. Sobre ellas, situadas al tresbolillo, se sitúan las del anillo siguiente, de tal manera que las impostas de unas bóvedas se apoyan sobre las claves de las inferiores.

Los senos de estas pequeñas bóveđas están macizados con objeto de absorber y compensar las fuerzas 
oblicuas y horizontales. El espesor del macizado se va reduciendo conforme la parábola de las bóvedas va ganando en esbeltez en el sentido ascendente de los sucesivos anillos.

El casquete esférico de cerramiento que se apoya en el anillo superior está construido con tres gruesos de rasilla común, que se empotran en la superficie que forman las bóvedas de dicho anillo.

Este peculiar sistema constructivo permite afirmar que se trata de una cúpula de estructura única, no de "una cúpula doble, con recintos interiores a través de dos capas perforadas" ni de una "estructura que sigue el sistema renacentista de doble cúpula" como hasta ahora la han descrito algunos autores.

En cuanto a los aspectos formales y decorativos de esta cúpula también conviene una precisión. Está demostrada la admiración que Gaudi sentía por la arquitectura hispano-musulmana, y en particular por la Alhambra de Granada. Algunos autores afirman que se inspiró en los baños de este conjunto andaluz para diseñar la cúpula del Palau Güell. Creo que un paralelo más racional - lo cual no quiere decir que Gaudi se inspirase necesariamente en él- es la cúpula de los baños de Yeni Kaplica en Bursa, Turquía, donde los tragaluces son circulares, la clave está practicada por un óculo cenital y en los paramentos verticales de la sala, existe un revestimiento cerámico de piezas hexagonales, elementos todos ellos que se repiten en el Palau.

\section{LA LINTERNA}

La linterna ocupa la parte media de la aguja, la zona donde adquiere una figura geométrica pura, un cono recto de planta circular. Este volumen arranca del último anillo escalonado de la cúpula, en cuya parte exterior se apoya abrazando dicho anillo y el casquete esférico que remata la cúpula.

En el interior de la linterna se encuentra un pequeño banco perimetral cuya función principal es ocultar las bóvedas del anillo mencionado y actuar como sumidero para evacuar a través de esas bóvedas las aguas de lluvia que pudieran penetrar en el recinto.

Fig. 4.-Detalle del vértice de la linterna. 1.-Revestimiento exterior. 2.-Estructura de la lintera. 3-Aberturas romboidales superiores. 4.-Viseras. 5.-Anclaje de la veleta a la obra. 6.-Cono de madera. 7.-Anclaje del árbol de la veleta a la base de madera. 8. - Anclaje de la parte externa de la base de la veleta a la obra. 9.-Abrazaderas metálicas de la base de la veleta. 10.-Arbol de la veleta. (Planimetría, Josep Maria Moreno; dibujo, Maite Gómez),

Fig. 4 bis.-Detalle del aro metálico intermedio de la linterna. 1.-Revestimiento exterior. 2-Estructura de cuatro gruesos de rasilla comủn. 3.-Aro metálico de descarga. 4.-Estructura de tres gruesos de rasilla común (Planimetria, Josep Maria Moreno; dibujo, Maite Gómez).
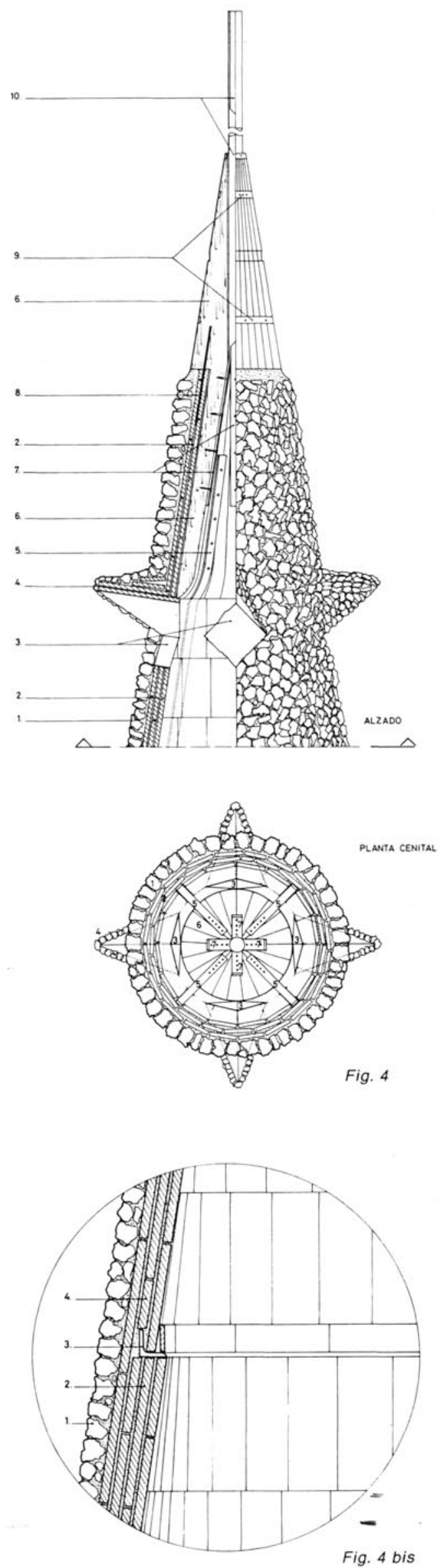

Fig. 4 bis 
Por encima del banco aparecen ocho ventanas de perfil parabólico que permiten la entrada de luz al interior del espacio central a través del óculo cenital de la misma cúpula. Una de estas ventanas, desprovista de antepecho, actúa como puerta de acceso al interior de la linterna y de comunicación con el resto de las azoteas mediante una pasarela.

Las ventanas están colocadas, a modo de buhardillas, en un plano vertical exterior respecto a la generatriz del cono. La separación entre la ventana vertical y el perfil inclinado del cono se salva mediante una bóveda que junto al revestimento de la superficie exterior alabeada ayudan a rigidizar el conjunto de la linterna.

Más arriba, según se asciende hacia el vértice del cono, aparecen doce aberturas romboidales, distribuidas al tresbolillo, formando cuatro grupos - opuestos dos a dos - de tres aberturas cada uno. Las aberturas del grupo superior están situadas al mismo nivel y las doce están protegidas por viseras de obra. La función de estas aberturas romboidales - que no tienen ningún tipo de cerramiento- es ventilar el espacio interior de la linterna, para mantener el equilibrio térmico con el ambiente exterior y evitar los movimientos de contracción-dilatación de la estructura.

\section{Sistema constructivo}

Las paredes de la linterna están construidas con material cerámico. Desde la base hasta la mitad de la altura, su espesor es de cuatro gruesos de rasilla común de $29 \times 14 \times 2 \mathrm{~cm}$, cambiando en este punto y hasta la base de la veleta a tres gruesos. Esta reducción de espesor facilita la esbeltez compositiva del interior del cono y lo aligera de un peso innecesario (Fig. 5). El vértice está construido con 2 gruesos y recubre parcialmente la base de la veleta.

Estructuralmente, la linterna se apoya en el último anillo de la cúpula, formando una base dentada que se empotra en las bóvedas abocinadas que componen dicho anillo. Más hacia arriba, un zuncho perimetral -construido con dos hiladas de ladrillo macizo cortado y aplantillado- da solidez a la fábrica y sirve de apoyo de las bóvedas de las ventanas, formando a su vez el antepecho de las mismas.

Las bóvedas parabólicas que forman las ventanas están construidas con tres gruesos de rasilla. La base de dichas bóvedas se apoya sobre el ancho de $10 \mathrm{~cm}$ del zuncho de ladrillo; el perfil exterior forma un plano vertical, y el interior se encastra, en un plano inclinado, con la superficie del cono.

A tres metros de la base de la linterna, en su punto medio y por encima de los ángulos superiores de las dos

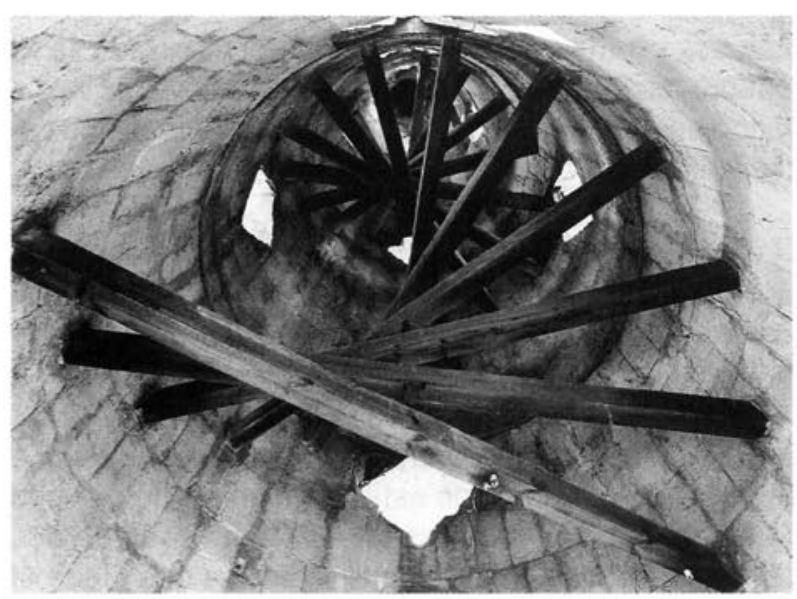

Fig. 5.-Travesaños de madera para absorber los empujes del vien. to. (Foto Jordi Isern, febrero de 1990).

primeras aberturas romboidales, un aro metálico de perfil $L$ de $6 \times 6 \mathrm{~cm}$, anclado en la fábrica, sirve para repartir y homogeneizar las cargas y para permitir el tránsito de cuatro a tres gruesos de rasilla.

Las viseras de las aberturas romboidales están construidas con dos gruesos de rasilla, formando dos triángulos unidos por el lado superior, que arrancan de los vértices laterales del rombo y recorren los lados superiores del mismo, encastrándose en éstos.

Por encima de los vértices superiores de las cuatro últimas aberturas romboidales, la estructura de la linterna se reduce a dos gruesos de rasilla. Por encima de este punto, sobre el primer grueso interior de rasilla, se asienta la base del cono hueco de madera de la veleta. Los dos gruesos de rasilla restantes siguen hacia arriba recubriendo externamente dicho cono de madera, hasta la mitad de su altura. El exterior de la linterna, como se explica más adelante, está revestido con piedra arenisca vidriada. Además de su papel estético, este revestimiento cumple la función -al estar vidriado - de proteger la estructura de las humedades de filtración ya que no absorbe agua por su propia naturaleza. Por el interior, la estructura carece de revestimiento, apareciendo visto el primer grueso de rasilla.

Los espesores de la estructura son, según hemos visto, de 10,7 y $5 \mathrm{~cm}$, que correponde a los gruesos de rasilla en las zonas de ventanas, aberturas romboidales y cono de la veleta, respectivamente. El revestimiento exterior de piedra tiene un espesor aproximado de $5 \mathrm{~cm}$.

En el interior de la linterna (Fig. 6), por encima de las ventanas, unos travesaños de madera colocados de forma diametral y superpuestos en espiral, se empotran en la estructura de fábrica con la misión de absorber los empujes externos del viento a qūe está sometida la esbelta aguja. 


\section{LA VELETA.PARARRAYOS}

La aguja está coronada por la veleta-pararrayos, un bello elemento decorativo y funcional digno complemento del conjunto (Figs. 7 y 8 ).

La parte inferior del elemento es una estructura de madera de forma compuesta - un tronco de cono y un cono sobre él-donde se ancla y soporta el árbol o eje principal de la veleta. El tronco de cono inferior es hue$\mathrm{co}$, arranca por encima de las cuatro aberturas superiores de la linterna y está embebido en la estructura de obra de ésta. El cono superior, el único que sobresale de la fábrica de la linterna, está forrado por otro cono que sirve de apoyo a una esfera de $60 \mathrm{~cm}$ de diá. metro, de la que nacen dieciséis puntas cónicas de diverso tamaño que señalan los puntos cardinales; las puntas mayores se orientan a los cuatro puntos principales y las menores a los secundarios. Los vértices de estas puntas funcionan además como pararrayos.

Por encima de estas estructuras aparece la veleta propiamente dicha, formada por una figura zoomórfica -un murciélago con las alas extendidas- que señala la dirección del viento y en el lado opuesto un pandero circular que cumple la doble función de vela y contrapeso. La veleta se corona con una cruz griega

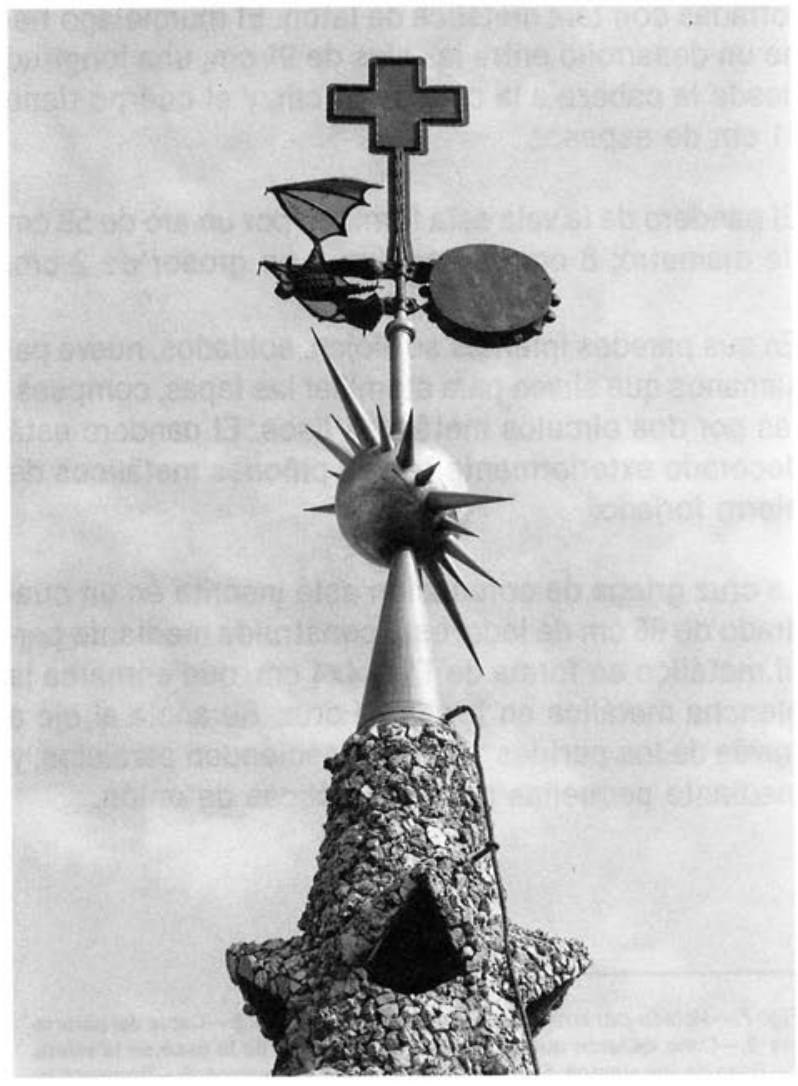

Fig. 6. - La veleta-pararrayos, poco después de ser restaurada. (Fo. to Jordi Isern, febrero de 1990). colocada sobre el eje que gira al unísono con el murciélago y el pandero.

La parte visible de la veleta tiene una altura de cinco metros y añadiendo la parte oculta en la fábrica de la linterna alcanza una longitud total de seis. El peso de la parte metálica es de $150 \mathrm{~kg}$.

\section{Sistema constructivo}

Estructuralmente (Fig. 5), la base de madera es la más importante del elemento pues actúa como muelle entre la veleta y la fábrica de la linterna donde se empo. tra. Tiene una altura total de $205 \mathrm{~cm}$ y geométricamente responde a una figura formada por un tronco de cono, la parte inferior, y un cono superpuesto, construidos ambos solidariamente.

El tronco de cono inferior -cuyos diámetros son 60 y $26 \mathrm{~cm}$ y su altura $105 \mathrm{~cm}$ - es hueco, formando unas paredes de $8 \mathrm{~cm}$ de espesor. Está construido mediante veintiún listones de madera ensamblados entre sí colocados de forma radial. A través de este hueco los anclajes de la veleta llegan a la estructura de obra de la linterna, donde se empotran. Se trata de cuatro patas metálicas de pasamano de $38 \times 8 \mathrm{~mm}$ con una longitud de $250 \mathrm{~cm}$. La parte superior de estos pasamanos es vista; tiene una longitud de $70 \mathrm{~cm}$, y está atornillada a la cara interna del cono de madera, mediante seis tornillos de cabeza cuadrada de los Ilamados de sierralima. Por la parte interna superior de este cono, aparece el extremo inferior del árbol metálico de la veleta y las cuatro patas formadas por pasamano de $55 \times 8$ centímetros de sección y $70 \mathrm{~cm}$ de longitud, fijadas cada una a la madera, mediante cuatro tornillos similares a los descritos anteriormente. Las patas inferiores que anclan el cono a la obra y las del árbol de la veleta, están situadas al tresbolillo y se sobrepasan entre si 25 $\mathrm{cm}$. Esta parte de la base de la veleta es la que está embebida en la obra de la linterna.

El cono superior es macizo; tiene una base de $44 \mathrm{~cm}$ de diámetro y $100 \mathrm{~cm}$ de altura. Por su eje pasa el árbol metálico de la veleta, al que abraza. En la corona circular que forma la superficie de la base de este co. no con el diámetro superior del tronco de cono inferior, se alojan cuatro anclajes metálicos de hierro forjado, que se empotran en la obra y en la madera. La parte empotrada en la obra tiene una sección rectangular de $10 \times 20 \mathrm{~mm}$ y la empotrada en el cono de madera es cilíndrica con un diámetro de $15 \mathrm{~mm}$.

Este cono superior sobresale por encima de la obra de la linterna y la sujeción de los listones que lo forman se consigue mediante abrazaderas metálic̄as torneadas y atornilladas que se encuentran embēbidas den. tro de la figura. Como se ha dicho, está forrado por un 


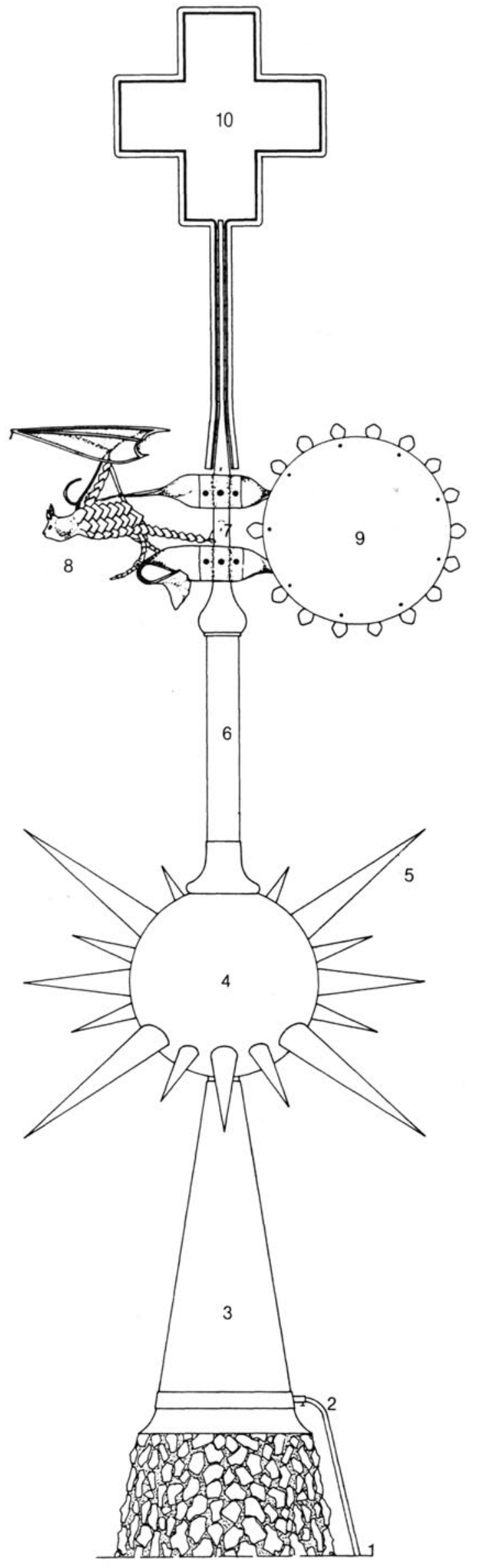

cono de latón dorado que lo protege de la intemperie y que sustenta la esfera que forma la rosa de los vientos y el pararrayos. Esta esfera, también de latón dorado, está construida con dos casquetes esféricos y un anillo que soporta las flechas de los vientos, cuyos vértices de cobre son las puntas Franklin del pararrayos. Todos estos elementos están soldados con estaño, a excepción de las puntas Franklin de cobre, que están sujetas al latón mediante roscas. Por encima de la esfera, una trompeta de latón invertida protege el árbol de la veleta.

Este árbol tiene un desarrollo total de $250 \mathrm{~cm}$ de altura y un diámetro exterior de $7 \mathrm{~cm}$. Los $50 \mathrm{~cm}$ superiores son huecos con un diámetro interior de $55 \mathrm{~mm}$ y un fondo cónico, donde se aloja el eje de la veleta cuyo extremo inferior acaba en punta cónica, para formar con el árbol el cojinete de giro de la veleta. Al eje se sujetan, mediante piezas de hierro forjado y tornillos, el murciélago y el pandero que hacen de veleta y vela respectivamente.

El murciélago es de hierro forjado y en su lomo tiene un lingote de plomo para contrapesar todo el conjunto. Las alas y la parte trasera superior del animal están forradas con tela metálica de latón. El murciélago tiene un desarrollo entre las alas de $91 \mathrm{~cm}$, una longitud desde la cabeza a la cola de $51 \mathrm{~cm}$, y el cuerpo tiene $11 \mathrm{~cm}$ de espesor.

El pandero de la vela esta formado por un aro de $58 \mathrm{~cm}$ de diámetro; $8 \mathrm{~cm}$ de anchura y un grosor de $2 \mathrm{~cm}$.

En sus paredes internas se alojan, soldados, nueve pasamanos que sirven para atornillar las tapas, compuestas por dos círculos metálicos lisos. El pandero está decorado exteriormente con 16 piñones metálicos de hierro forjado.

La cruz griega de coronación está inscrita en un cuadrado de $66 \mathrm{~cm}$ de lado; está construida mediante perfil metálico en forma de T de $4 \times 4 \mathrm{~cm}$, que enmarca la plancha metálica en forma de cruz. Se ancla al eje a través de los perfiles "T", que descienden paralelos, y mediante pequeñas piezas metálicas de unión.

Fig. 7.-Veleta-pararrayos. 1.-Vértice de la linterna. 2.-Cable del pararrayos. 3.-Cono de latón que protege la parte exterior dela base de la veleta 4.-Rosa de los vientos. 5.-Puntas Franklin del pararrayos. 6.-Trompeta in vertida que protege el árbol de la veleta. 7.-Eje de latveleta. 8.-Murciélago 9.-Pandero. 10.-Cruz griega de remate. (Planimetrá, Josep Maria Moreno; dibujo, Maite Gómez). 


\section{FUNCIONAMIENTO ESTÁTICO DEL CONJUNTO}

La estructura de la aguja (Fig. 4) se sustenta y descarga en dos paredes de piedra, paralelas a las fachadas, y en las dos jácenas metálicas de un primer zunchado, paralelas a las medianeras, que se empotran en dichas paredes y que reducen su luz mediante columnas y machones, también de piedra. Estas jácenas y paredes son las que delimitan el perímetro de la sala central de la planta noble del Palau Güell.

Por debajo de la cuarta planta del edificio, ocho ménsulas de piedra colocadas perpendicularmente entre si, dos a dos, en los ángulos del cuadrado de la sala, de forma que los extremos de sus voladizos sean tangentes, sirven de apoyo al segundo zunchado de cuatro jácenas metálicas, que a su vez reducen el cuadrado de la planta. En los vértices de este nuevo cuadrado y por encima de las jácenas se asientan cuatro pilares que atraviesan, ascendiendo, la cuarta planta, que por el interior de ésta son circulares y por el espacio de la sala aparecen como paralelepipedos, formando una curva parabólica con la dirección de las diagonales de la planta de forma que cuando alcanzan su máxima altura se han convertido en otras cuatro ménsulas. Uniendo figuradamente los extremos de estas ménsulas, obtendremos un tercer cuadrado inferior a los anteriores, y es en esta última figura donde se halla inscrita la circunferencia de la cúpula.

De los extremos interiores de estas ménsulas - hacia la sala- arrancan las impostas de los cuatro arcos de la cúpula; en cada uno de los extremos exteriores se apoyan - por encima del techo de la cuarta plantacinco viguetas de perfil metálico, dispuestas en abanico, que forman la base de apoyo de los arcos tangentes y abocinados, paralelos a los de la cúpula y cuya función estática es doble; por un lado, contrarrestan los empujes horizontales de la cúpula, haciendo de contrafuertes de ésta; y por otro, sirven de contrapeso al momento de vuelco, que tienen, por su morfología, las ménsulas que soportan los arcos de la cúpula. Estos arcos abocinados emergen por encima de la azotea en forma de gajos.

Los cuatro arcos parabólicos superiores están unidos entre si por pechinas, que transforman el cuadrado de su base en la circunferencia en la que se asienta la cú-
Fig. 8.-Desarrollo estático-constructivo de la cúpula y la aguja del Palau Güell.

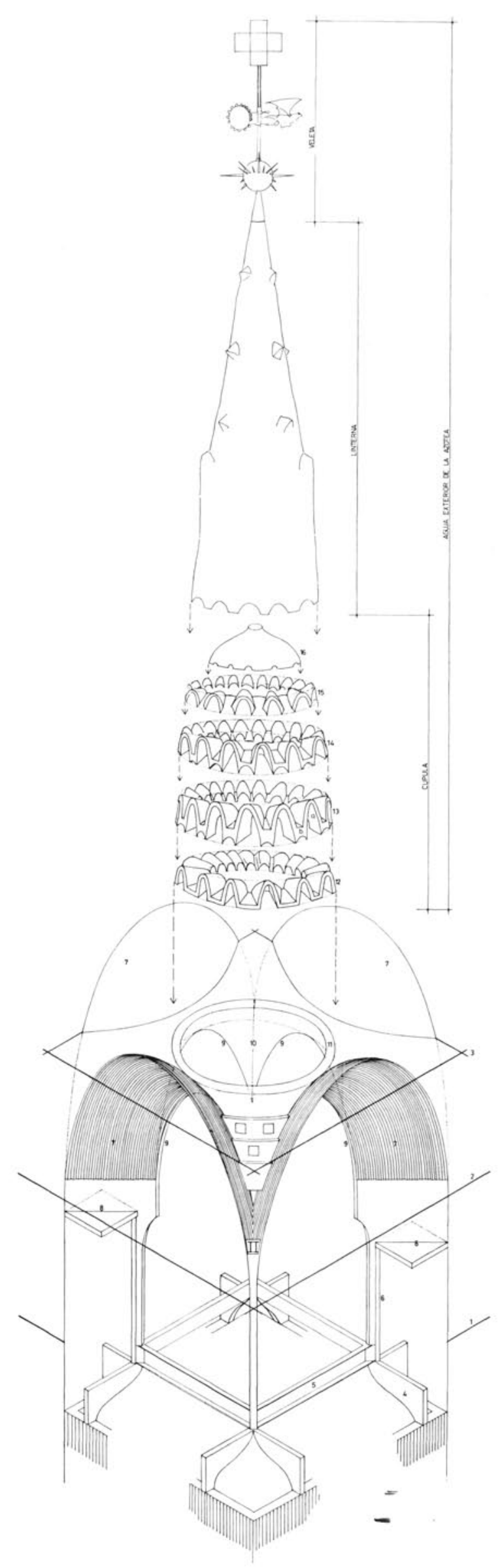

http://informesdelaconstruccion.revistas.csic.es 
pula. Ésta, como ya se ha dicho anteriormente, está construida mediante sesenta y cuatro pequeñas bóvedas abocinadas, superpuestas y concéntricas, colocadas en cuatro hiladas o anillos de dieciséis unidades cada uno. La distribución de estas bóvedas y el macizado de sus senos, crean una situación de empotramiento y equilibrio perfecto, compensándose todas las fuerzas opuestas que se escapan de las estructuras.

El casquete esférico que cierra la cúpula, y la linterna que lo cubre, tienen el perímetro de la base de forma dentada como consecuencia de la superficie donde se asientan (bóvedas del último anillo superior de la cúpula), creando también un empotramiento perfecto.

De lo analizado anteriormente, puede deducirse que los elementos que forman la aguja del Palau Güell están fuertemente empotrados entre si y forman una estructura hiperestática.

\section{EL REVESTIMIENTO EXTERIOR DE LA AGUJA}

Para revestir el exterior de la aguja - tanto la linterna como la parte de la cúpula que emerge de la azotea-, Gaudi utilizó un material singular: pequeños fragmentos de rocas areniscas rojas vitrificadas procedentes de las paredes internas de hornos de cal de leña amortizados (Fig. 9).

Esta roca -formada en el Triásico inferior - la conocemos comúnmente como piedra de amolar y la usaban los afiladores en el ejercicio de su oficio. Si la sometemos a cocción intermitente, a una temperatura entre 900 y $1.000{ }^{\circ} \mathrm{C}$, se funde y vitrifica la superficie que está en contacto con el fuego.

Conforme se van repitiendo los ciclos de cocción, la roca va sufriendo alteraciones químicas en su composición, de manera que si en el primer ciclo se obtiene un vidriado verdoso sobre el fondo rojo de la roca, en el ciclo número treinta aproximadamente, tanto el vidriado como lo roca se ha convertido de color blanco y ha aumentado su dureza.

Siguiendo la repetición de los ciclos por encima de los treinta y cinco, la roca se quema y gasifica convirtiéndose en una escoria negra, porosa y deforme, que Gaudi también utilizó en otras obras, como en el revestimiento exterior de la cripta de la Colonia Güell.

Las rocas utilizadas en el Palau Güell provienen del macizo del Garraf, cuyo limite por el sur se extiende junto a la costa de la provincia de Barcelona, entre los caseríos de Castelldefels y Sitges. Este macizo, que por el este limita con el estuario del rio Llobregat, está formado geológicamente por tres tipos de rocas superpuestos. El estrato inferior se compone de pizarras

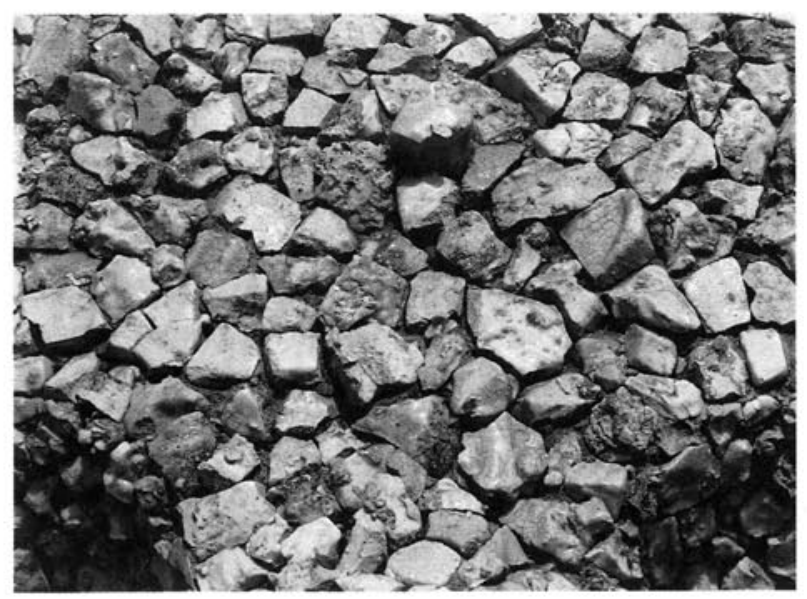

Fig. 9.-Fragmentos de roca arenisca vitrificada usada como revestimiento de la linterna del Palau Güell. (Foto Jordi Isern, febrero 1990).

primarias, que son la continuación del plegamiento existente en la sierra de Collcerola y que forma el lecho del río Llobregat. Este estrato es bien visible en los pueblos ribereños del río, como Gavà, Viladecans, Sant Boi y Sant Vicenç dels Horts.

El estrato intermedio está formado por rocas areniscas rojas del Triásico inferior, que son las utilizadas en el revestimiento que aqui estudiamos. El estrato es visible en los barrancos del macizo, que abarcan desde el castillo de Aramprunyá en Gavà, hasta el pueblo de Torrelles de Llobregat.

El estrato superior está formado por rocas calizas margosas del Triásico medio, y abarca la parte alta del macizo, afectando en esta zona a los municipios de Begues, Olesa de Bonesvalls y Vallirana. Son las rocas de este estrato las que se utilizaron en la construcción de las fachadas y otros elementos del Palau Güell.

El estrato superior del macizo del Garraf es óptimo para la fabricación de cal y de cemento. Por ello, antiguamente era la producción de cal la industria principal de la zona y su obtencion se efectuaba en los hornos construidos según la tradición romana (Fig. 10). Las paredes de estos hornos, de unos veinte centímetros de espesor, se construian con las mismas piedras calizas, lo cual comportaba que en cada hornada perdian por calcinación $2 \mathrm{~cm}$ de espesor y, en consecuencia, el horno aumentaba en 4 su diámetro. Repitiendo el proceso de cocción cuatro o cinco veces, las paredes del horno se debilitaban de tal manera que, una vez amortizado, debia abandonarse y construir otro nuevo.

Con el tiempo se descubrió, tal vez fortuitamente, que revistiendo la cara interior de las paredes del horno con bloques de la piedra arenisca citadā, ésta permitía la reutilización del horno unas treinta y dos veces, hasta 
Informes de la Construcción, Vol. 42 n. ${ }^{\circ} 408$, julio/agosto, 1990

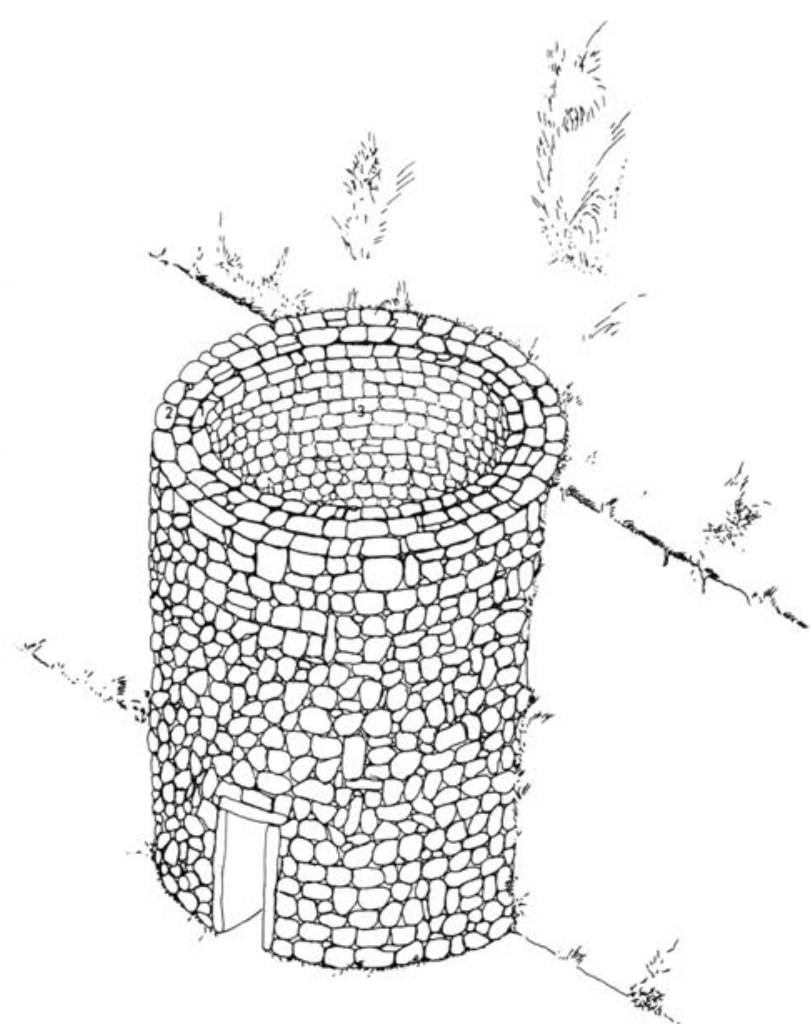

Fig. 10.- Horno de cal de tradición romana en el macizo de Garraf. (Dibujo, Txetxu Sanz).

que los efectos de vitrificación descritos anteriormente llegaban a su límite y aconsejaban la construcción de un nuevo horno, por amortización del primero. Esto permitió la utilización del horno durante cuatro años, a razón de ocho hornadas anuales, antes de que éste se abandonase por amortizado. Fue en estos hornos donde Gaudi encontró el material utilizado como revestimiento de la linterna del Palau Güell (Fig. 11).

Con la aparición del cemento y la crisis de la primera guerra mundial, la cal pasó a ser un aglomerante secundario en la construcción y su producción desapareció como industria familiar y artesanal, transformándose en una industria mecánica con fábricas a pie de cantera, con cintas transportadoras, hornos continuos y cadenas de envasado.

Los pocos caleros que subsistieron, de los cuales todavía queda uno en los bosques de Vallirana, tuvieron que apurarse para rentabilizar y amortizar al máximo el tipo de horno utilizado, y lo consiguieron sustituyendo el forro de piedra arenisca por otro de ladrillo refractario, consiguiendo resultados definitivos con un mantenimiento mínimo consistente en la reparación, de vez en cuando, de los ladrillos refractarios degradados.

Con el desarrollo tecnológico e industrial de nuestro siglo, otro problema que han debido afrontar los cale-

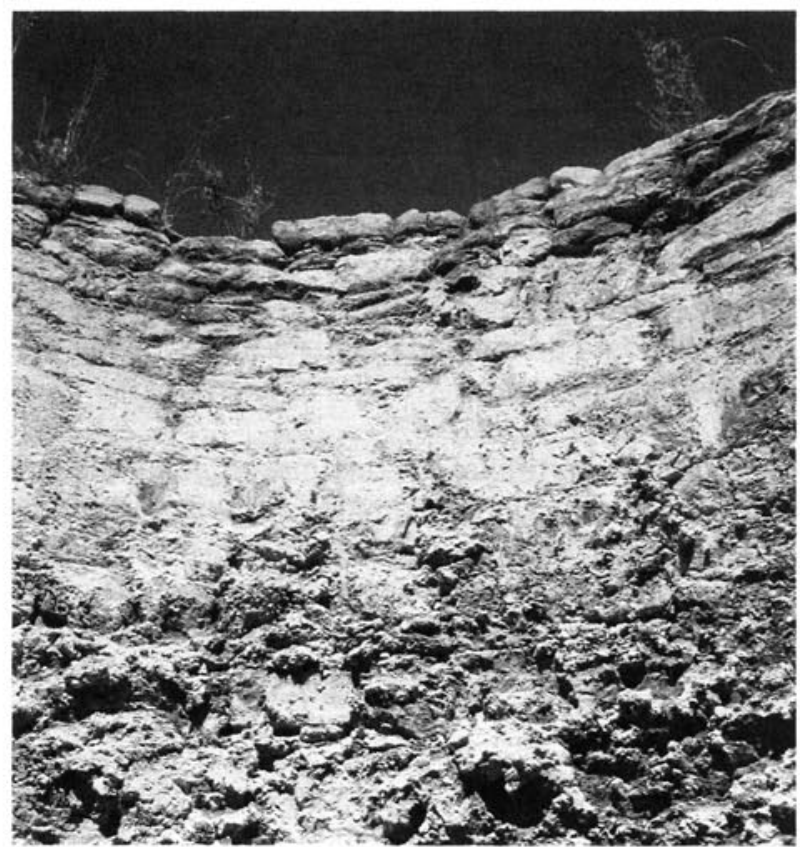

Fig. 11.-Interior de un horno de cal amortizado en el macizo de Garraf. (Foto Josep María Moreno, septiembre de 1988).

ros ha sido la dificultad de encontrar mano de obra para limpiar los bosques del matorral, que era el combustible utilizado. En la actualidad, el horno de Vallirana, todavia en uso, utiliza como combustible neumáticos de goma de rueda de automóvil. La cal que producen los pocos caleros en activo, es altamente apreciada por su pureza y se utiliza sobre todo en la fabricación de estucos decorativos y enlucidos (1).

Gaudi utilizó también como acabado decorativo en la linterna del Palau Güell los azulejos fragmentados (trencadís), que revisten los intradoses de las pequeñas bóvedas abocinadas del primer anillo, entre las carpinterias que las cierran y el perfil exterior de las mismas (Fig. 12).

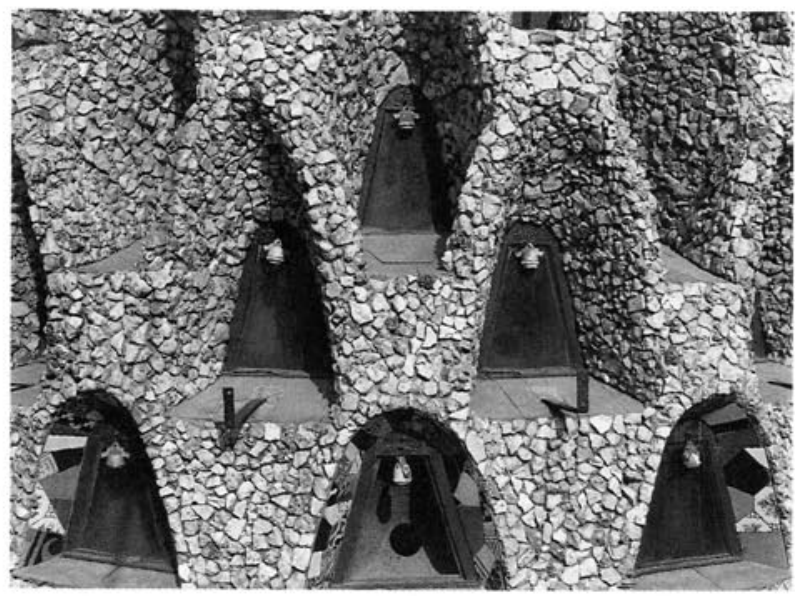

Fig. 12.-Revestimiento de azulejo troceado en el imtradós de las hornacinas de la base de la linterna y restos de la iluminación artificial moderna. (Foto Jordi Isern, febrero de 1990). 


\section{LA ILUMINACIÓN DEL CONJUNTO}

La iluminación natural del interior del edificio desde la azotea se consigue a través de la linterna, mediante el óculo cenital de la cúpula y de los pequeños tragaluces circulares que se distribuyen por la superficie de ésta. La luz también penetra por los óculos de las lunetas y por los ventanales que dan a la planta de buhardillas, hasta donde llega la luz a través de las claraboyas de la azotea.

La iluminación artificial se consigue actualmente mediante electricidad, con bombillas de incandescencia. La peculiaridad de la instalación y la falta de documentación respecto a la fecha de montaje y puesta en funcionamiento, no nos permite precisar si se trata de una modificación de una instalación previa proyectada por Gaudi.

Por los restos de instalaciones encontrados en el interior del edificio, se sabe que éste estuvo originalmente alumbrado con lámparas de gas, pero no hay que olvidar que en 1884 la Compañía Canadiense iluminó Ia Rambla de Barcelona con electricidad, de forma experimental. Es posible, pues, que el palacio poseyera instalación eléctrica casi desde su inauguración.
Respecto a los materiales elétricos que se encuentran en la aguja se puede decir que los pasamuros y aisladores de porcelana, parecen anteriores a 1926, pero no hemos podido precisar su fecha de instalación.

Como conclusión de este análisis, la hipótesis más verosímil que puede hacerse con los datos de que disponemos actualmente es que la aguja del Palau Güell se iluminó eléctricamente entre 1913 y 1926, con un tendido aéreo a base de dos hilos separados; esta primera instalación, tal vez por obsoleta, debió ser sustituida por la actual entre 1950-55 y la alimentación aérea a base de cable forrado de plástico antihumedad, debió ser colocada en las reparaciones efectuadas en el edificio entre 1970-74. Queda por tanto la duda razonable de que Gaudi previera la iluminación artificial del interior a través de la azotea, y en todo caso cuál fue el sistema instalado.

(1) Expreso desde estas lineas mi agradecimiento a los señores Joan Farreras, de Vallirana y Xavier Artisó, presidente en 1988 del Gremio de Fabricantes de Cal de Barcelona, por su apreciada colaboración durante la investigación de esta parte del trabajo.

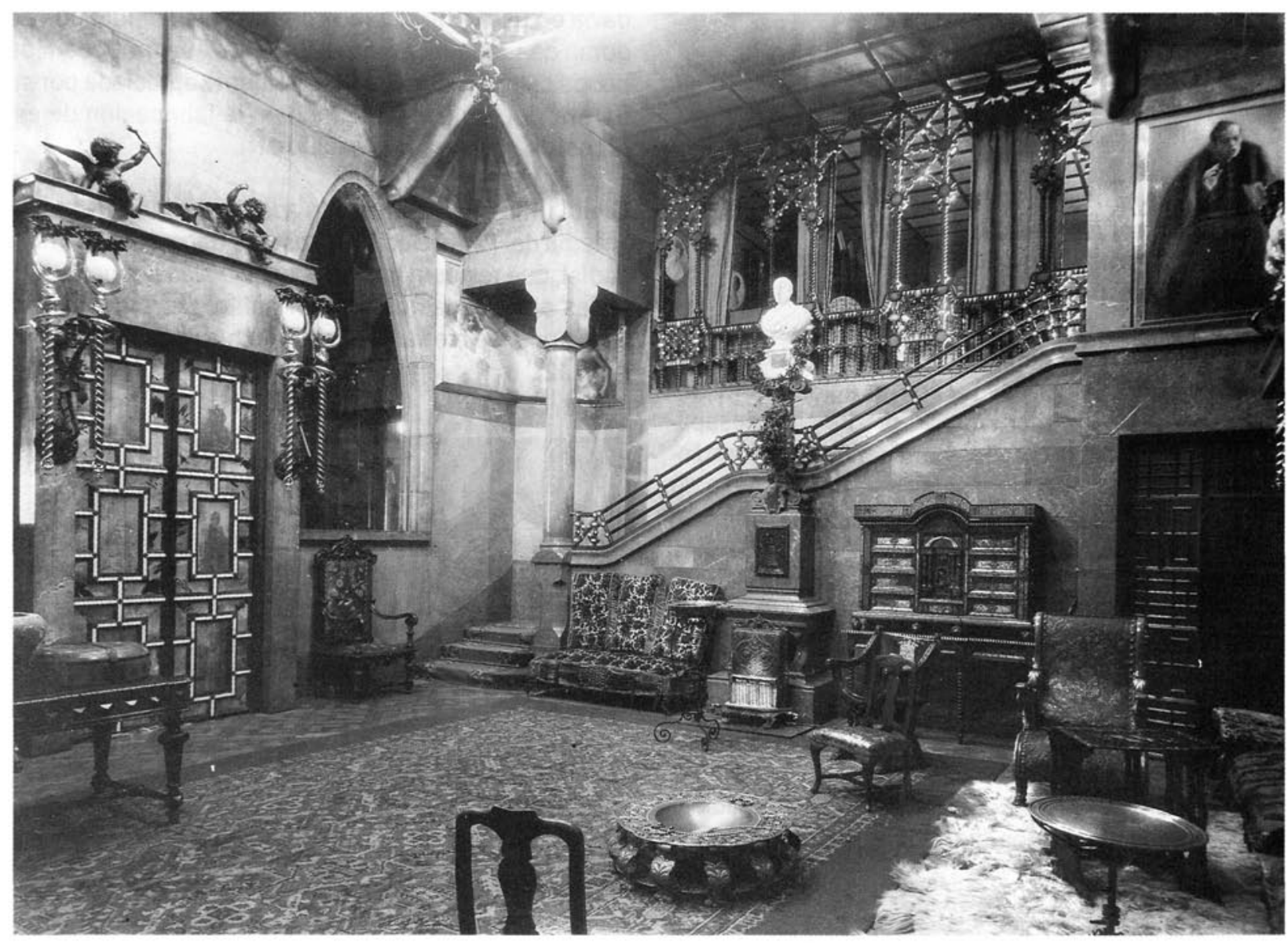

Fig. 13.-Salón-capilla iluminado cenitalmente por la cúpula-linterna. (Foto Arxiu Mas, 1932). 